\title{
A New Lifetime Exponential-X Family of Distributions with Applications to Reliability Data
}

\author{
Xiaoyan Huo, ${ }^{1}$ Saima K. Khosa, ${ }^{2}$ Zubair Ahmad (D), ${ }^{3}$ Zahra Almaspoor (D), \\ Muhammad Ilyas, ${ }^{4}$ and Muhammad Aamir ${ }^{5}$ \\ ${ }^{1}$ Yatai School of Business Management, Jilin University of Finance and Economics, Changchun, Jilin Province, China \\ ${ }^{2}$ Department of Statistics, Bahauddin Zakariya University, Multan, Pakistan \\ ${ }^{3}$ Department of Statistics, Yazd University, P.O. Box 89175-741, Yazd, Iran \\ ${ }^{4}$ Department of Statistics, University of Malakand, Dir (L), Chakdara, Khyber Pakhtunkhwa, Pakistan \\ ${ }^{5}$ Department of Statistics, Abdul Wali University Mardan, Mardan, Khyber Pakhtunkhwa, Pakistan
}

Correspondence should be addressed to Zubair Ahmad; z.ferry21@gmail.com and Zahra Almaspoor; zahra.ferry21@gmail.com

Received 16 March 2020; Revised 7 May 2020; Accepted 17 June 2020; Published 18 August 2020

Academic Editor: Giovanni Falsone

Copyright ( 2020 Xiaoyan Huo et al. This is an open access article distributed under the Creative Commons Attribution License, which permits unrestricted use, distribution, and reproduction in any medium, provided the original work is properly cited.

\begin{abstract}
Modeling reliability data with nonmonotone hazards is a prominent research topic that is quite rich and still growing rapidly. Many studies have suggested introducing new families of distributions to modify the Weibull distribution to model the nonmonotone hazards. In the present study, we propose a new family of distributions called a new lifetime exponential- $X$ family. A special submodel of the proposed family called a new lifetime exponential-Weibull distribution suitable for modeling reliability data with bathtub-shaped hazard rates is discussed. The maximum-likelihood estimators of the model parameters are obtained. A brief Monte Carlo simulation study is conducted to evaluate the performance of these estimators. For illustrative purposes, two real applications from reliability engineering with bathtub-shaped hazard functions are analyzed. The practical applications show that the proposed model provides better fits than the other nonnested models.
\end{abstract}

\section{Introduction}

tThe hazard rate function (also known as failure rate function) is an important reliability characteristic. It deals with the failure of the system at the time, say $t$, given that the system has not failed prior to time $t$. Among the hazard rate functions, the bathtub-shaped hazard rate curve is well known in reliability engineering. It represents the failure behavior of various engineering systems having initially a decreasing failure rate during the very first phase, a relatively constant failure rate in the middle part of the life (usually called useful life period), and finally an increasing failure rate in the last phase. In the context of the reliability theory, these three phases are known, respectively, as burning, random, and wear-out failure regions.

In the last two decades, many new life distributions capable of modeling data with the bathtub-shaped hazard rate function (hrf) have been introduced in the literature. Most of them are the modifications and extensions of the two-parameter Weibull distribution. For example, a three-parameter exponentiated Weibull (EW) of Mudholkar and Srivastava [1] has a bathtub-shaped hazard function. A three-parameter modified Weibull extension (MWEx) of Xie et al. [2] exhibits data modeling with bathtub shape. The truncated Weibull distribution of Zhang and Xie [3] has a bathtub-shaped hazard function. The two-parameter flexible Weibull extension (FWEx) of Bebbington et al. [4] has increasing, decreasing, or bathtub-shaped function. An interesting extension of the Weibull model called Zubair-Weibull (ZW) distribution proposed by Ahmad [5] is capable of modeling the data exhibiting the bathtub-shaped failure rate. A threeparameter extended alpha power transformed Weibull (EAPTW) of Ahmad et al. [6] and a three-parameter new extended alpha power transformed Weibull (NEAPTW) of Ahmad et al. [7] are all having failure rate function that can be increasing, decreasing, or bathtub-shaped. 
The parametric models such as the exponential, Rayleigh, Weibull, lognormal, and gamma distributions have been extensively used in fitting biomedical and reliability data [8]. The researchers in reliability engineering and other related fields have shown a great interest in studying the failure time of electronic devices [9]. An appropriate parametric model is always of interest in survival and reliability analysis, as it provides a concise description of the characteristics of failure times as well as hazard function that may not be available with nonparametric methods [10]. Also, as observed in Zhu et al. [8], the parametric Weibull is more flexible distribution than the Cox semiparametric model [11], since the associated hazard rate is not constant over time. However, unfortunately, the Weibull model is not capable of modeling data with nonmonotonic (unimodal, modified unimodal, and bathtub-shaped) hrf.

Due to the importance of the statistical distributions in reliability engineering and other related fields, the researchers are motivated to introduce new flexible distributions. In this regard, serious attempts have been made and still growing rapidly. The new developments have been made through many different approaches such as (i) transformation of variables, (ii) composition of two or more distributions, (iii) compounding of distributions, and (iv) finite mixture of distributions.

Recent studies of Bagnato and Punzo [12] showed that the transformation approach is simple to use, but most often the inference and computation of the other distributional characteristics become complicated.

Another promising approach for obtaining new families of distributions, which gives a reasonably good fit for reallife data, is the method of composition; see Bakar et al. [13]. However, it should be noted that the new distributions obtained by the composition approach involve more than three parameters causing difficulties in the estimation process and computational efforts are required.

Another prominent approach is compounding of distributions to cater data modeling with unimodality. However, the density obtained via this method may not have a closed-form expression which makes the estimation more cumbersome as shown in Punzo et al. [14].

Finite mixture models represent a further approach to define very flexible distributions which are also able to capture, for instance, multimodality of the underlying distribution (Punzo et al. [15]). The price to pay for this greater flexibility is a more complicated and computationally challenging inference.

Using the above approaches, a number of families of distributions have been introduced; see Topp-Leone odd log-logistic family [16], the Burr- $X$ generator [17], exponentiated transmuted-G family [18], the Burr-XII system of densities [19], a new extended G family [20], type I general exponential class of distributions [21], and type II general exponential class of distributions [22], among others.

Carrying out this branch of distribution theory, Alzaatreh et al. [23] defined the T- $X$ family method to introduce new families of distributions. Let $v(t)$ be the probability density function (pdf) of a random variable, say $T$, where
$T \in[m, n], \quad-\infty \leq m<n<\infty$, and let $W[F(x ; \xi)]$ be a function of $F(x ; \xi)$ of a random variable, say $X$, satisfying the following conditions:

(1) $W[F(x ; \xi)] \in[m, n]$

(2) $W[F(x ; \xi)]$ is differentiable and monotonically increasing

(3) $W[F(x ; \xi)] \longrightarrow m \quad$ as $\quad x \longrightarrow-\infty \quad$ and $W[F(x ; \xi)] \longrightarrow n$ as $x \longrightarrow \infty$

The cdf of the T-X family of distributions is defined as follows:

$$
G(x)=\int_{m}^{W[F(x ; \xi)]} v(t) \mathrm{d} t, \quad x \in \mathbb{R},
$$

where $W[F(x ; \xi)]$ satisfies the conditions stated above. The pdf corresponding to (1) is given by

$$
g(x)=\left\{\frac{\partial}{\partial x} W[F(x ; \xi)]\right\} v\{W[F(x ; \xi)]\}, \quad x \in \mathbb{R}
$$

For the contributed work based on the idea of T- $X$ approach, we refer to Ahmad et al. [24]. Using the approach of $\mathrm{T}-X$ method, one can introduce new members of survival family [25] via the cdf:

$$
G(x)=1-\int_{m}^{W[\bar{F}(x ; \xi)]} v(t) \mathrm{d} t, \quad x \in \mathbb{R},
$$

where $\bar{F}(x ; \xi)=1-F(x ; \xi)$ is the survival function of the baseline distribution.

Taking inspiration from (1), in this article, we propose a new family of distributions to provide the best fit to data in reliability sciences and other related fields. Let $T \sim \exp (1)$, then its cdf is given by

$$
V(t)=1-e^{-t}, \quad t \geq 0 .
$$

The density function corresponding to (4) is

$$
v(t)=e^{-t}, \quad t>0 \text {. }
$$

If $v(t)$ follows (5) and setting $W[F(x ; \xi)]=-\log ((1-$ $\left.F(x ; \xi)) / e^{F(x ; \xi)}\right)$ in (1), Ahmad et al. [26] defined the cdf of the weighted $\mathrm{T}-X$ family as follows:

$$
G(x ; \xi)=1-\left(\frac{1-F(x ; \xi)}{e^{F(x ; \xi)}}\right), \quad x \in \mathbb{R},
$$

where $\xi$ is the parameter vector that may belong to $\mathbb{R}$. Ahmad et al. proposed an extended version of (6), called Z-family of distributions by setting $W[F(x ; \xi)]=-\log \left\{(1-F(x ; \xi)) / \beta^{F(x ; \xi)}\right\}$ and $v(t)=e^{-t}$, in (1), given by

$$
G(x ; \beta, \xi)=1-\left\{\frac{1-F(x ; \xi)}{\beta^{F(x ; \xi)}}\right\}, \quad \beta>0, x, \xi \in \mathbb{R} .
$$

The expression in (7) represents a wide family of univariate continuous distributions. Clearly, when $\beta=1$, the cdf of the proposed family derived in (7) becomes identical to the baseline cdf, and for $\beta=e$, the expression (7) reduces to (6). The pdf corresponding to (7) is given by 


$$
g(x ; \beta, \xi)=f(x ; \xi)\left(\frac{1+(\log \beta)[1-F(x ; \xi)]}{\beta^{F(x ; \xi)}}\right), \quad x \in \mathbb{R} .
$$

No doubt, the Z-family defined in (8) is a more flexible class of distributions. However, expression (8) involves the logarithmic term resulting in complication of the density function and the derivation of many mathematical properties becomes complicated. To avoid this problem, we introduce an alternative form of (8). The newly proposed family is introduced by reparametrizing $\beta=e^{\theta}$. The form of the newly proposed family is much simple and flexible than the Z-family.

If $T$ follows (5) and setting $W[F(x ; \xi)]=-\log \left((1-F(x ; \xi)) / e^{\theta F(x ; \xi)}\right)$ in (1), we define the cdf of the new lifetime exponential- $X$ (NLTE- $X$ ) family by

$$
G(x ; \theta, \xi)=1-\left(\frac{1-F(x ; \xi)}{e^{\theta F(x ; \xi)}}\right), \quad \theta>0, x \in \mathbb{R} .
$$

Clearly, for $\theta=1$, expression (9) reduces to (6). The density function and hrf corresponding to (9) are, respectively, given by

$$
\begin{aligned}
& g(x ; \theta, \xi)=\frac{f(x ; \xi)}{e^{\theta F(x ; \xi)}}\{1+\theta \bar{F}(x ; \xi)\}, \quad x \in \mathbb{R}, \\
& h(x ; \theta, \xi)=\frac{f(x ; \xi)}{1-F(x ; \xi)}\{1+\theta \bar{F}(x ; \xi)\}, \quad x \in \mathbb{R} .
\end{aligned}
$$

A special submodel of the proposed family called a new lifetime exponential-Weibull (NLTE-W) distribution is considered in detail. The proposed model is very flexible and offers data modeling with increasing, decreasing, and bathtub- and inverted bathtub-shaped hazard rate functions. Finally, we concentrate our attention to illustrate the proposed model by analyzing two real-life applications from reliability engineering.

The paper is outlined as follows: a special submodel of the proposed family is defined in Section 2. Some mathematical properties are derived in Section 3. Maximumlikelihood estimation of the model parameters is addressed in Section 4. Two real-life applications to reliability data are analyzed in Section 5. Finally, some concluding remarks are presented in Section 6.

\section{Special Submodels}

This section offers some special submodels of the proposed class.

2.1. NLTE-Weibull Distribution. Let $F(x ; \xi)$ be the cdf of the Weibull model given by $F(x ; \xi)=1-e^{-\gamma x^{\alpha}}, x \geq 0, \alpha, \gamma>0$, where $\xi=(\alpha, \gamma)$. Then, the cdf of the NLTE-W distribution has the following expression:

$$
G(x ; \alpha, \gamma, \theta)=1-\left(\frac{e^{-\gamma x^{\alpha}}}{e^{\theta\left(1-e^{-\gamma \alpha^{\alpha}}\right)}}\right), \quad x \geq 0 \alpha, \gamma, \theta>0 .
$$

The pdf and hrf of the NLTE-W model are given, respectively, by

$$
\begin{aligned}
& g(x ; \alpha, \gamma, \theta)=\frac{\alpha \gamma x^{\alpha-1} e^{-\gamma x^{\alpha}}}{e^{\theta\left(1-e^{-\gamma x^{\alpha}}\right)}}\left(1+\theta e^{-\gamma x^{\alpha}}\right), \quad x>0, \\
& h(x ; \alpha, \gamma, \theta)=\alpha \gamma x^{\alpha-1}\left(1+\theta e^{-\gamma x^{\alpha}}\right), \quad x>0 .
\end{aligned}
$$

For different values of the model parameters, plots of the density function of the NLTE-W model are sketched in Figure 1.

For different values of the model parameters, plots of the hrf of the NLTE-W are sketched in Figures 2 and 3.

In Figure 1, we plotted different shapes for the density of NLTE-W distribution for fixed values of $\gamma$ and different values of $\alpha$ and $\theta$. When $\alpha<1$, the proposed model behaves like exponential distribution. But, as the value of $\alpha$ and $\theta$ increases, the proposed model captures the characteristics of the Weibull distribution. However, the proposed model has certain advantages over the Weibull distribution. For example, it is capable of modeling lifetime data with monotonic and nonmonotonic hrf. As it is given in Figures 2 and 3, the hrf of the proposed model is very flexible in accommodating different shapes, namely, decreasing, increasing, unimodal, and most importantly with bathtubshaped hrf. Hence the NLTE-W distribution becomes an important model to fit several real lifetime data in applied areas such as reliability engineering and biomedical analysis.

2.2. NLTE-Rayleigh Distribution. Let $F(x ; \gamma)$ be the cdf of the Rayleigh model given by $F(x ; \gamma)=1-e^{-\gamma x^{2}}, x \geq 0, \gamma>0$. Then, the cdf of the NLTE-Rayleigh (NLTE-R) distribution has the following expression:

$$
G(x ; \gamma, \theta)=1-\left(\frac{e^{-\gamma x^{2}}}{e^{\theta\left(1-e^{-\gamma x^{2}}\right)}}\right), \quad x \geq 0, \gamma, \theta>0 .
$$

The pdf and hrf of the NLTE-R distribution are given, respectively, by

$$
\begin{aligned}
& g(x ; \gamma, \theta)=\frac{2 \gamma x e^{-\gamma x^{2}}}{e^{\theta\left(1-e^{-\gamma x^{2}}\right)}}\left(1+\theta e^{-\gamma x^{2}}\right), \quad x>0, \\
& h(x ; \gamma, \theta)=2 \gamma x\left(1+\theta e^{-\gamma x^{2}}\right), \quad x>0 .
\end{aligned}
$$

For different values of the model parameters, plots of the density and hazard rate functions of the NLTE-R model are sketched in Figure 4.

2.3. NLTE-Exponential Distribution. Let $F(x ; \gamma)$ be the cdf of the exponential model given by $F(x ; \gamma)=1-e^{-\gamma x}, x \geq 0, \gamma>0$. Then, the cdf of the NLTEexponential (NLTE-E) distribution has the following expression:

$$
G(x ; \gamma, \theta)=1-\left(\frac{e^{-\gamma x}}{e^{\theta\left(1-e^{-\gamma x}\right)}}\right), \quad x \geq 0, \gamma, \theta>0 .
$$




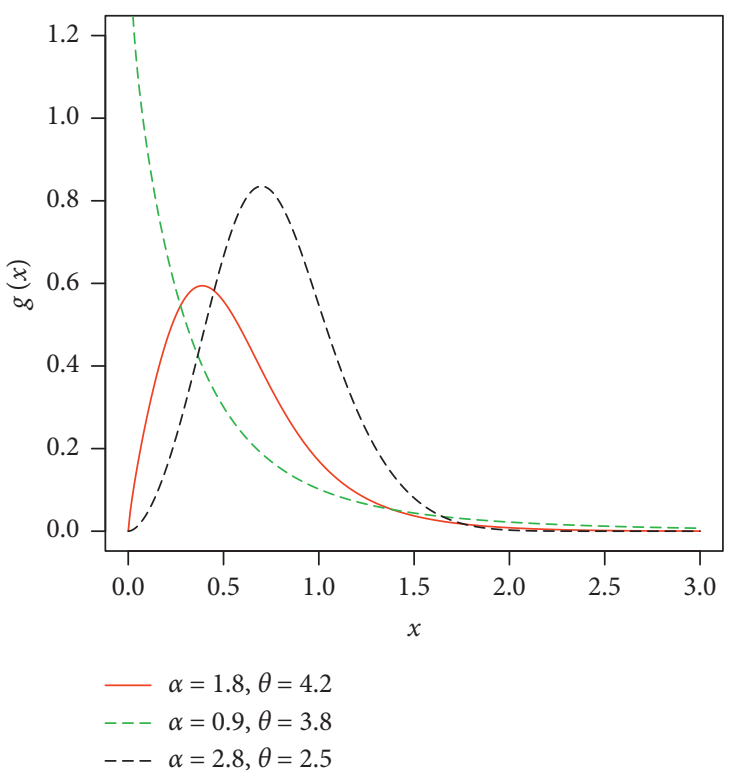

(a)

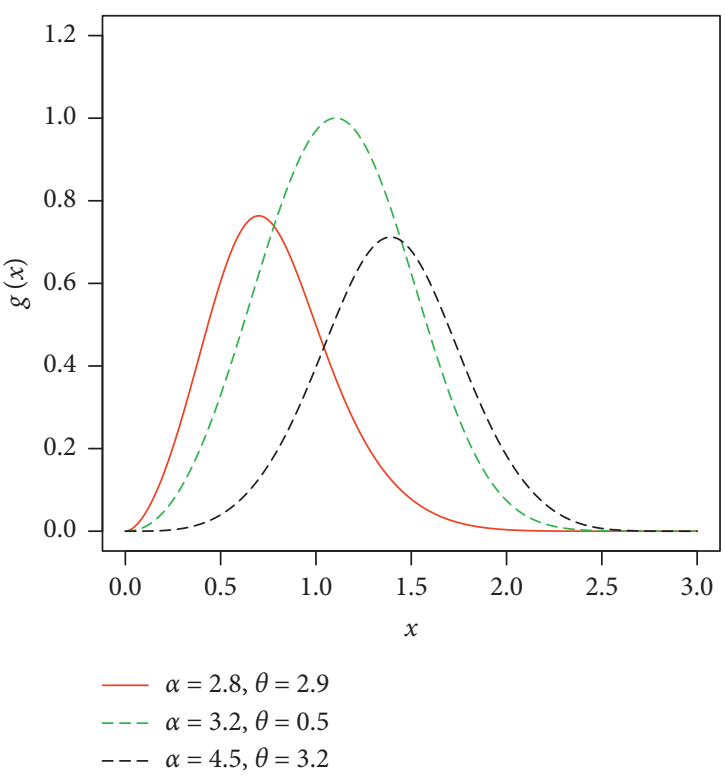

(b)

FIgURE 1: Different plots for the density function of the NLTE-W distribution.

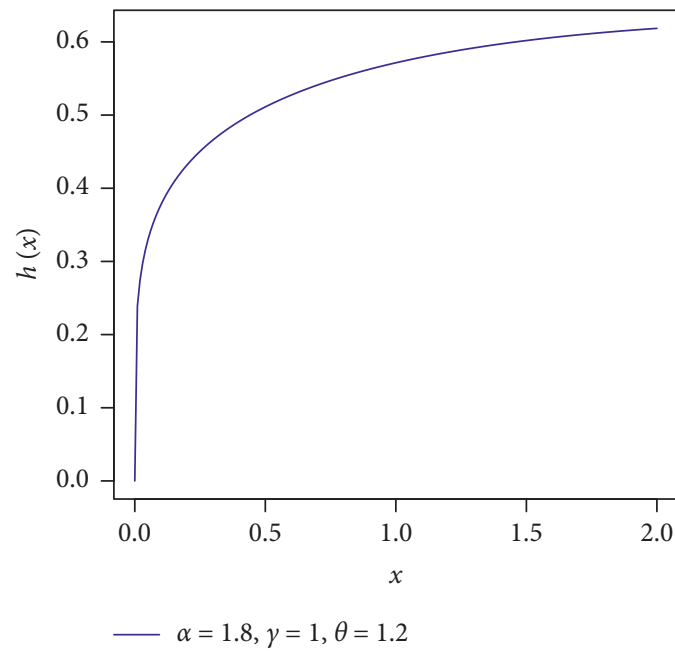

(a)

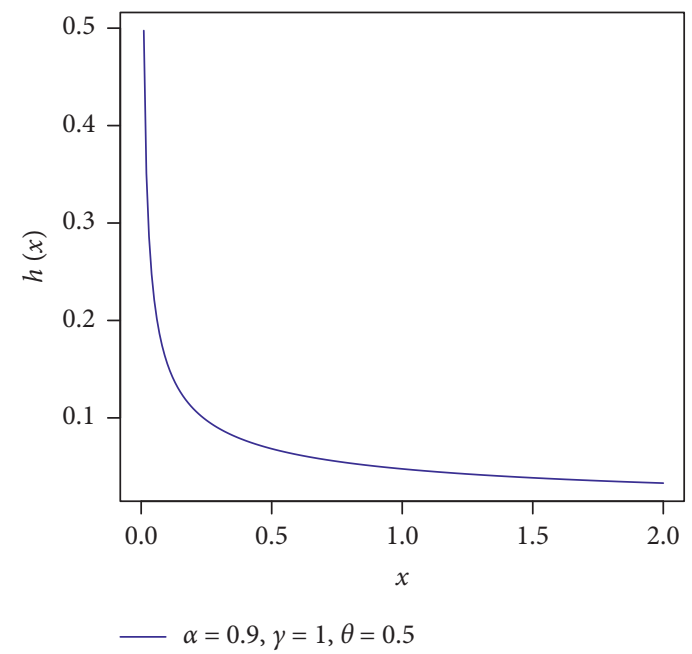

(b)

FIGURE 2: Increasing and decreasing plots for the hrf of the NLTE-W distribution.

The pdf and hrf of the NLTE-E model are given, respectively, by

$$
\begin{aligned}
& g(x ; \gamma, \theta)=\frac{\gamma e^{-\gamma x}}{e^{\theta\left(1-e^{-\gamma x}\right)}}\left(1+\theta e^{-\gamma x}\right), \quad x>0, \\
& h(x ; \gamma, \theta)=\gamma\left(1+\theta e^{-\gamma x}\right), \quad x>0 .
\end{aligned}
$$

For different values of the model parameters, plots of the density function of the NLTE-E distribution are sketched in Figure 5.

In Figure 5, we plotted different shapes for the density of NLTE-E distribution for different values of $\gamma$ and $\theta$. From Figure 5, we can see that the hrf of the NLTE-E distribution behaves approximately constant. However, the proposed model has certain advantages over the exponential distribution. For example, it is capable of modeling lifetime data with increasing hrf where the classical exponential distribution is not capable of modeling data with increasing hrf. Hence, the NLTE-E distribution becomes an important model to fit several real lifetime data in applied areas such as reliability engineering and biomedical analysis, where modeling data with increasing hrf are of interest.

\section{Mathematical Properties}

In this section, some mathematical properties of the NLTE$X$ distributions are derived. 


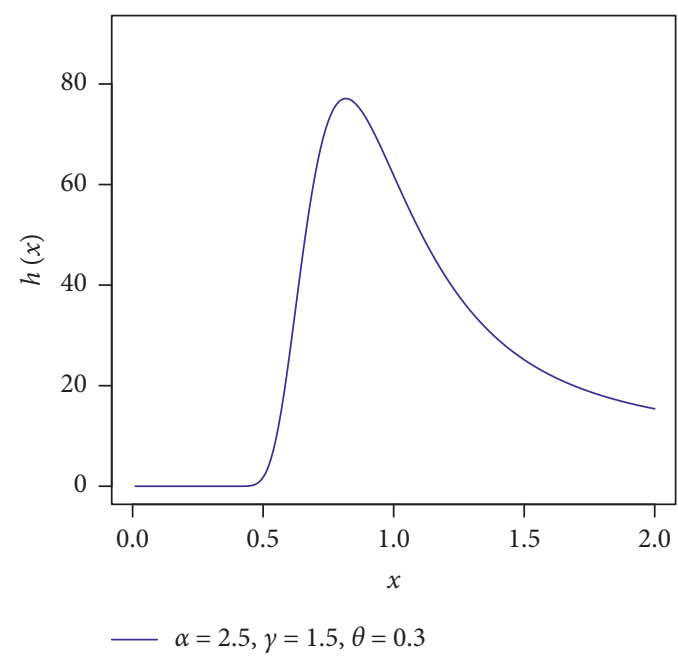

(a)

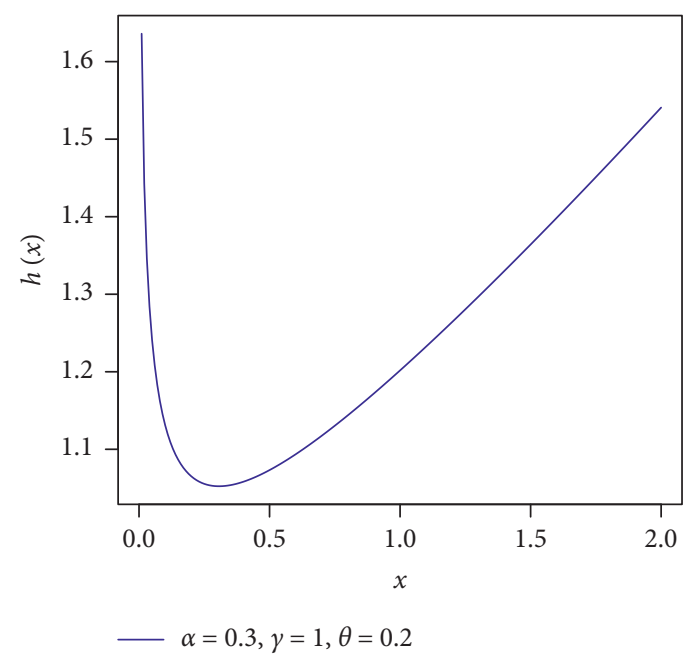

(b)

FIgURE 3: Unimodal and bathtub-shaped hrf of the NLTE-W distribution.

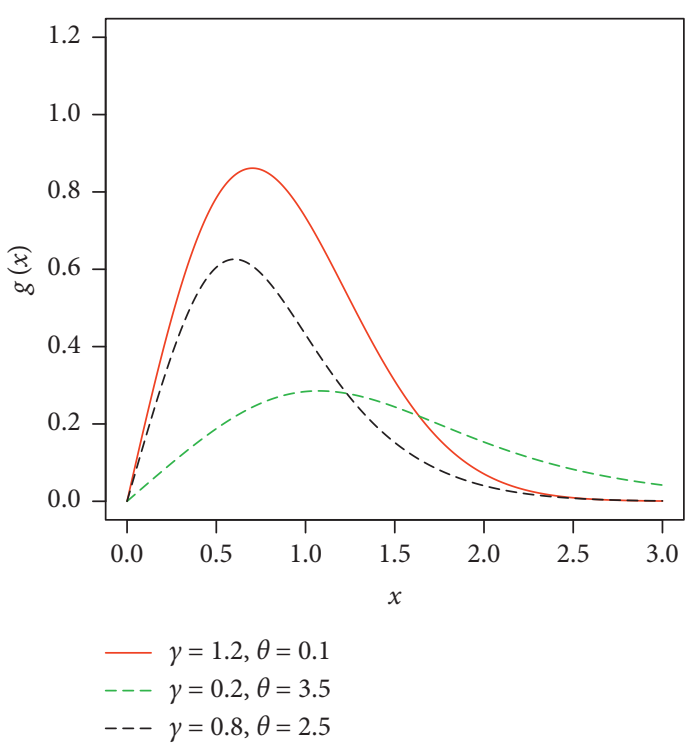

(a)

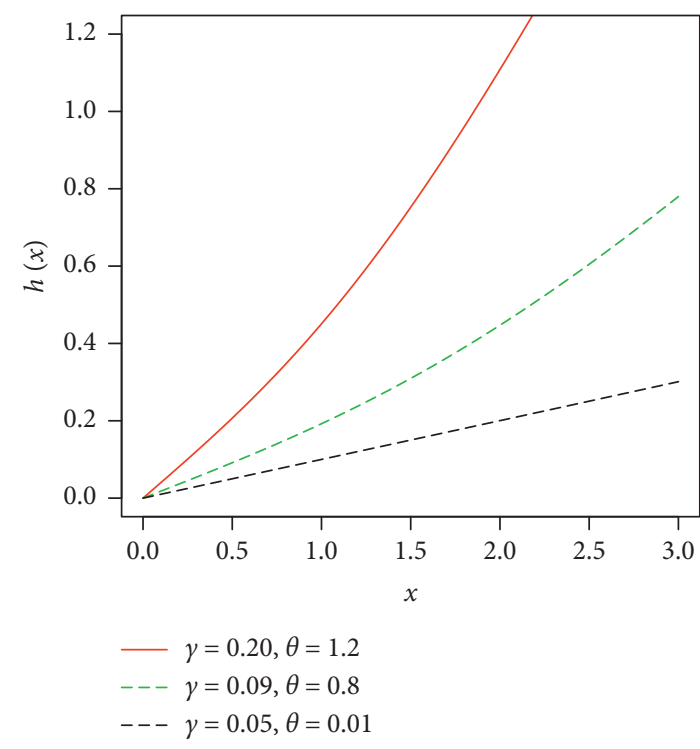

(b)

FIgURE 4: Different plots for the density and hazard rate functions of the NLTE-R distribution.

3.1. Quantile Function. The quantile function of NLTE- $X$ family is derived as follows:

$$
x=Q(u)=G^{-1}(u)=F^{-1}(t),
$$

where $t$ is the solution of the equation $(1-u) e^{\theta F(x ; \xi)}+F(x ; \xi)-1$ and $u$ has the uniform distribution on interval $(0,1)$. Nonlinear expression (18) can be used to obtain the random numbers for the NLTE- $X$ family of distributions.

3.2. Moments. Here, we derive some kinds of moments for the NLTE- $X$ family. For the sake of simplicity, we omit the dependency of $g(x ; \xi)$ and $G(x ; \xi)$ on the parameter vector $\xi$. Density (8) can be represented as follows:

$$
\begin{aligned}
& g(x)=\sum_{i=0}^{\infty} \frac{(-1)^{i}}{i !} \theta^{i} f(x ; \xi) F(x ; \xi)^{i}[1+\theta \bar{F}(x ; \xi)], \\
& g(x)=\sum_{i=0}^{\infty} \frac{(-1)^{i}}{i !}\left[\theta^{i} f(x ; \xi) F(x ; \xi)^{i}+\theta^{i+1} f(x ; \xi) F(x ; \xi)^{i+1}\right], \\
& g(x)=\sum_{i=0}^{\infty} \frac{(-1)^{i}}{i !} \tau_{i},
\end{aligned}
$$

where $\tau_{i}=\theta^{i} f(x ; \xi) F(x ; \xi)^{i}+\theta^{i+1} \sum_{j=0}^{1}(-1)^{j}\left(\begin{array}{c}n \\ j\end{array}\right) f(x ; \xi)$ $F(x ; \xi)^{i+j+1}$. For any positive integer $r$, the $r^{\text {th }}$ moment of the NLTE- $X$ distributions is given by 


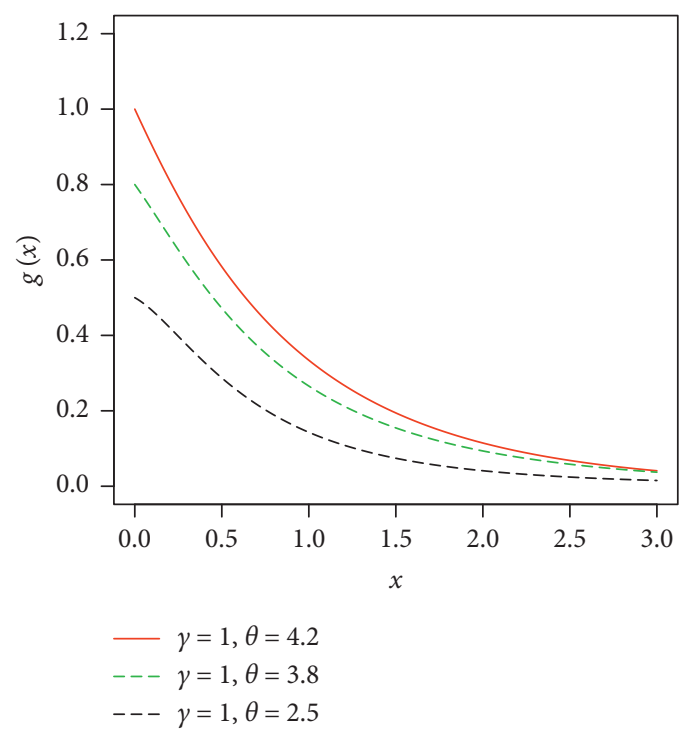

(a)

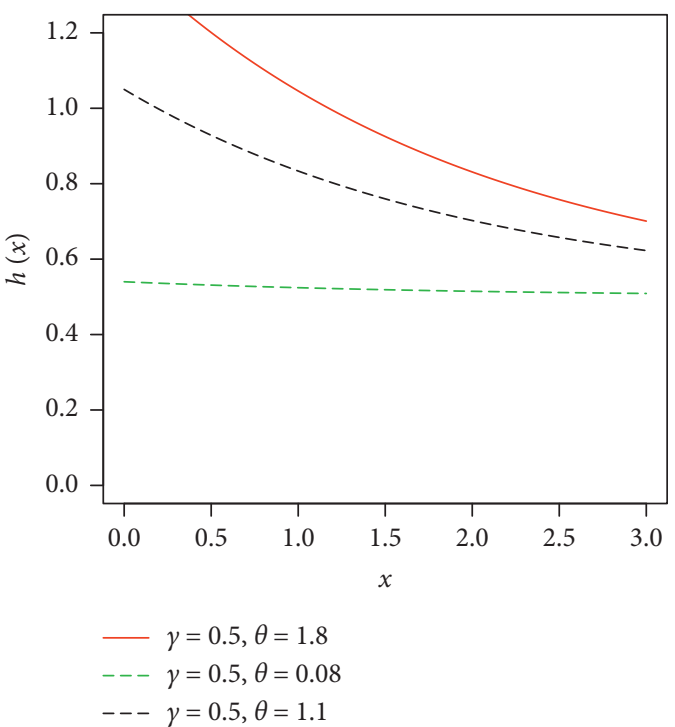

(b)

FIGURE 5: Different plots for the density function of the NLTE-E distribution.

$$
\mu_{r}^{\prime}=E\left(X^{r}\right)=\int_{-\infty}^{\infty} x^{r} g(x) \mathrm{d} x
$$

On using (20) in (21), we get the $r^{\text {th }}$ moment of the NLTE- $X$ distributions.

For $r=1,2,3,4$, we get the first four moments of the NLTE- $X$ distributions. The effects of the shape parameters on the skewness and kurtosis can be detected on the moments. Based on moments, we obtain skewness and kurtosis measures of the NLTE-Weibull distribution. The skewness of NLTE-Weibull distribution is obtained using the following expression:

$$
\text { skewness }=\frac{\mu_{3}}{\mu_{2}^{3 / 2}}
$$

where $\mu_{2}$ and $\mu_{3}$ are the second and third moments of the random variable $X$ with pdf (8). Furthermore, the kurtosis of $X$ is derived as follows:

$$
\text { kurtosis }=\frac{\mu_{4}}{\mu_{2}^{2}}
$$

where $\mu_{4}$ is the fourth moment of $X$. These measures are less sensitivity to outliers. Plots for the mean, variance, skewness, and kurtosis of the NLTE-Weibull distribution are displayed in Figure 6.

3.3. Residual Life. The residual life plays a vital role in practice particularly in reliability theory. The remaining waiting time for an event to happen conditioned that we have already been waiting is what we call residual life. Let $X$ follow NLTE- $X$ family of distributions, then the residual life of $X$ is given by

$$
\begin{aligned}
& \epsilon=\frac{S(x+t)}{S(x)}, \\
& \epsilon=\frac{[1-F(x+t)] e^{\theta F(x)}}{[1-F(x)] e^{\theta F(x+t)}} .
\end{aligned}
$$

3.4. Reverse Residual Life. The reverse residual life of a lifetime random variable is of interest in many areas of applied sciences such as survival analysis, actuarial studies, and risk management. The residual lifetime of $X$ denoted by $\bar{\epsilon}(x)$ is given by

$$
\begin{aligned}
& \bar{\epsilon}(x)=\frac{S(x-t)}{S(x)} \\
& \bar{\epsilon}(x)=\frac{[1-F(x-t)] e^{\theta F(x)}}{[1-F(x)] e^{\theta F(x-t)}} .
\end{aligned}
$$

3.5. On Other Means and Moments. The following result proposes an expansion of the primitive:

$$
\int_{-\infty}^{t} x^{r} g(x) \mathrm{d} x=\sum_{i=0}^{\infty} \frac{(-1)^{i}}{i !} \tau_{r, i},
$$

where $\tau_{r, i} \int_{-\infty}^{t} x^{r} \tau_{i} \mathrm{~d} x$.

Several crucial conditional moments can be obtained using the integral $\int_{-\infty}^{t} x^{r} g(x) \mathrm{d} x$ for various values of $r$. The most useful of them are presented as follows:

(i) The $r^{\text {th }}$ conditional moments of $X$ are given by 


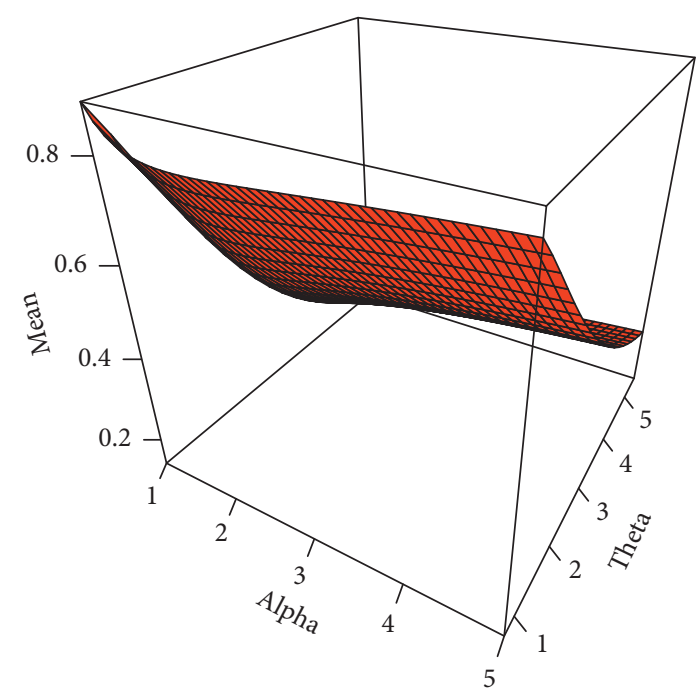

(a)

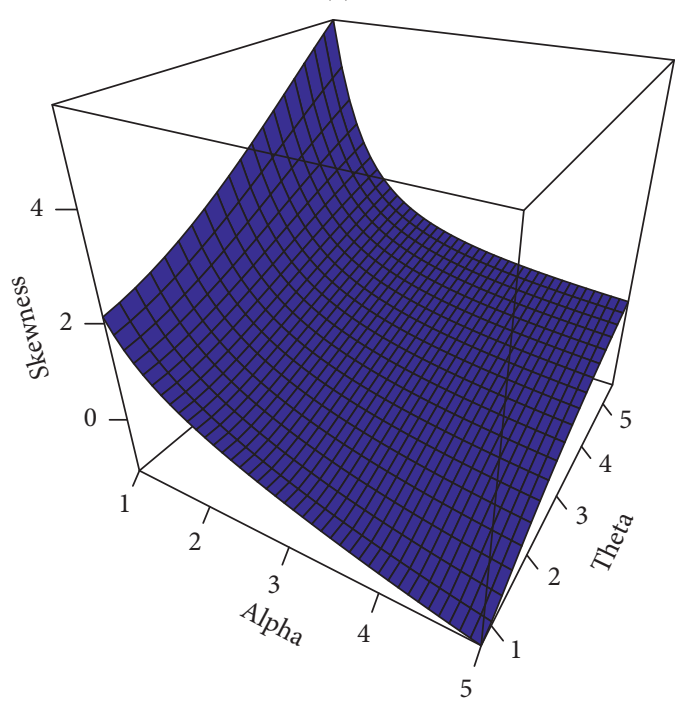

(c)

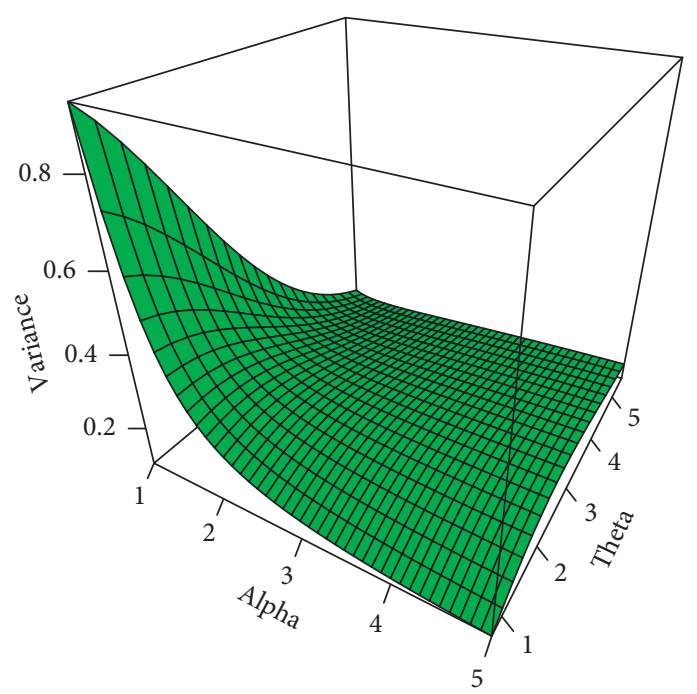

(b)

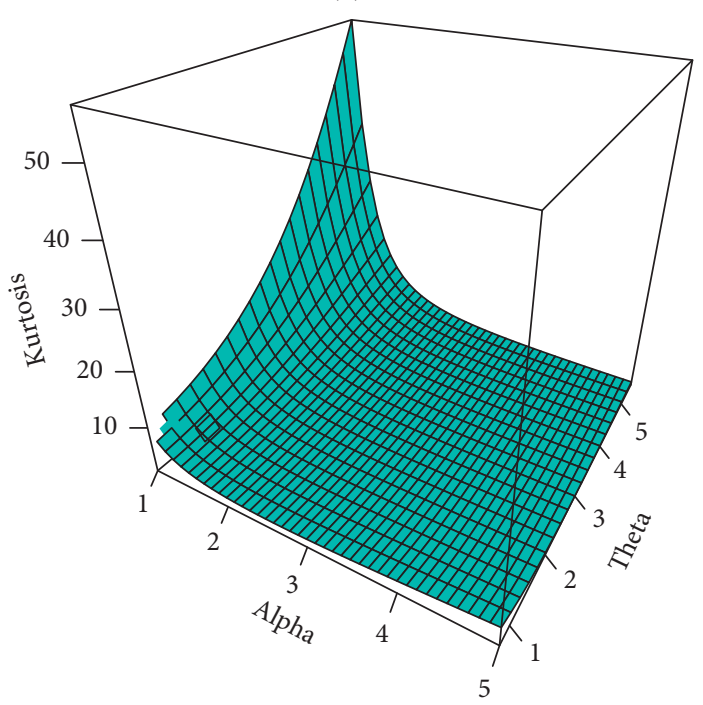

(d)

FIGURE 6: Plots of mean, variance, skewness, and kurtosis of the NLTE-W distribution.

$$
\begin{aligned}
E\left(X^{r} \mid X>t\right) & =\frac{1}{1-G(t)} \int_{t}^{+\infty} x^{r} g(x) \mathrm{d} x \\
& =\frac{1}{1-G(t)}\left(E\left(X^{r}\right)-\int_{-\infty}^{t} x^{r} g(x) \mathrm{d} x\right) .
\end{aligned}
$$

(ii) The $r^{\text {th }}$ reversed moments of $X$ are given by

$$
E\left(X^{r} \mid X \leq t\right)=\frac{1}{G(t)} \int_{-\infty}^{t} x^{r} g(x) \mathrm{d} x
$$

(iii) The mean deviations of $X$ about the mean, say $\mu$, are given by

$$
\delta=E(|X-\mu|)=2 \mu G(\mu)-2 \int_{-\infty}^{\mu} x g(x) \mathrm{d} x,
$$

where $\mu=E(X)$.

(iv) The mean deviations of $X$ about the median, say $M$, are given by

$$
\tau=E(|X-M|)=\mu-2 \int_{-\infty}^{M} x g(x) \mathrm{d} x .
$$

The residual life parameters can be also determined using $E\left(X^{r}\right)$ and $\int_{-\infty}^{t} x^{r} g(x ; \theta, \eta) \mathrm{d} x$ for several values of $r$. In particular, the following

(i) The mean residual life is defined as follows: 
$K(t)=E(X-t \mid X>t)=\frac{1}{S(t)}\left(E(X)-\int_{-\infty}^{t} x g(x) \mathrm{d} x\right)-t$,

and the variance residual life is given by

$$
\begin{aligned}
V(t)= & \operatorname{Var}(X-t \mid X>t) \\
= & \frac{1}{S(t)}\left(E\left(X^{2}\right)-\int_{-\infty}^{t} x^{2} g(x) \mathrm{d} x\right)-t^{2} \\
& -2 t K(t)-[K(t)]^{2} .
\end{aligned}
$$

(ii) The mean reversed residual life is defined as follows:

$$
L(t)=E(t-X \mid X \leq t)=t-\frac{1}{G(t)} \int_{-\infty}^{t} x g(x) \mathrm{d} x
$$

and the variance reversed residual life is defined as

$$
\begin{aligned}
W(t)= & \operatorname{Var}(t-X \mid X \leq t)=\frac{1}{G(t)} \int_{-\infty}^{t} x^{2} g(x) \mathrm{d} x \\
& +2 t L(t)-[L(t)]^{2}-t^{2} .
\end{aligned}
$$

\section{Maximum-Likelihood Estimation and Monte Carlo Simulation Study}

In this section, we use the maximum-likelihood method to estimate the model parameters and also provide a Monte Carlo (MC) simulation study to assess the behavior of these estimators.

4.1. Maximum-Likelihood Estimation. In this section, we obtain the maximum-likelihood estimators (MLEs) of the model parameters of the NLTE- $X$ distributions from complete samples only. Let $x_{1}, x_{2}, \ldots, x_{n}$ be an observed sample of size $n$ obtained from (9). The corresponding loglikelihood function can be expressed as follows:

$$
\begin{aligned}
L\left(x_{i}, \theta, \xi\right)= & \sum_{i=1}^{n} \log f\left(x_{i}, \xi\right)+\sum_{i=1}^{n} \log \left[1+\theta \bar{F}\left(x_{i}, \xi\right)\right] \\
& -\theta \sum_{i=1}^{n} F\left(x_{i}, \xi\right) .
\end{aligned}
$$

The log-likelihood function can be maximized either directly or by solving the nonlinear likelihood function obtained by differentiating (34). We used the goodness-of-fit function optim() R-function with the argument method= "L-BFGS-B" to obtain the MLEs. The first-order partial derivatives of (34) with respect to the parameters are given, respectively, by

$$
\begin{aligned}
\frac{\partial}{\partial \theta} L\left(x_{i}, \theta, \xi\right)= & \sum_{i=1}^{n} \frac{\bar{F}\left(x_{i} ; \xi\right)}{\left[1+\theta \bar{F}\left(x_{i} ; \xi\right)\right]}-\sum_{i=1}^{n} F\left(x_{i} ; \xi\right) \\
\frac{\partial}{\partial \xi} L\left(x_{i}, \theta, \xi\right)= & \sum_{i=1}^{n} \frac{\partial f\left(x_{i} ; \xi\right) / \partial \xi}{f\left(x_{i} ; \xi\right)}-\theta \sum_{i=1}^{n} \frac{\partial F\left(x_{i} ; \xi\right) / \partial \xi}{\left[1+\theta \bar{F}\left(x_{i} ; \xi\right)\right]} \\
& -\theta \sum_{i=1}^{n} \frac{\partial}{\partial \xi} F\left(x_{i} ; \xi\right) .
\end{aligned}
$$

Setting $\partial / \partial \theta \log L\left(x_{i}, \theta, \xi\right)$ and $\partial / \partial \xi \log L\left(x_{i} ; \theta, \xi\right)$ equal to zero and solving numerically these expressions simultaneously yield the MLEs of $(\theta, \xi)$.

4.2. Simulation Study. In this section, we perform a Monte Carlo simulation study with the objective to assess the behavior of the MLEs of NLTE-W model via the optim() $R$ function with the argument method = "L-BFGS-B." It is used for maximizing the log-likelihood function of a probabilistic model. We consider $750 \mathrm{MC}$ replicates under different sample sizes $n=25,50, \ldots, 750$. For each sample size, we compute the average MLEs, mean square errors (MSE), biases, and absolute biases. The results obtained after performing the MC simulation are provided in Tables 1 and 2 and displayed graphically in Figures 7 and 8 .

The simulation results provided in Figures 7 and 8 indicate the following:

(i) The estimates are quite stable and, more importantly, are close to the true values for these sample sizes

(ii) The estimated biases decrease when the sample size $n$ increases

(iii) The estimated MSEs decay toward zero when the sample size $n$ increases

\section{Applications of the NLTE-W Model to Reliability Data}

In this section, we use two reliability datasets to illustrate the importance and flexibility of the proposed distribution. The comparison of the proposed distribution is made with a nested model, the Weibull distribution, and with some other nonnested models such as exponentiated Weibull (EW), alpha power transformed Weibull (APTW), transmuted Weibull (TW), and flexible Weibull extended (FWE) distributions. The analysis is performed via the optim() $R$ function with the argument method= "BFGS". The cdfs of the competing distributions are as follows:

(i) Weibull distribution

$$
G(x ; \alpha, \gamma)=1-e^{-\gamma x^{\alpha}}, \quad x \geq 0, \alpha, \gamma>0 .
$$

(ii) EW distribution 
TABLE 1: Simulation results of NLTE-W distribution.

\begin{tabular}{|c|c|c|c|c|}
\hline \multicolumn{5}{|c|}{ Set $1: \alpha=0.9, \theta=1.4$, and $\gamma=0.6$} \\
\hline$n$ & Parameters & MLE & MSEs & Biases \\
\hline \multirow{3}{*}{25} & $\widehat{\alpha}$ & 0.9545 & 0.0611 & 0.0545 \\
\hline & $\widehat{\theta}$ & 2.4888 & 3.8729 & 1.0888 \\
\hline & $\widehat{\gamma}$ & 0.7748 & 0.2732 & 0.1748 \\
\hline \multirow{3}{*}{50} & $\widehat{\alpha}$ & 0.9327 & 0.0271 & 0.0427 \\
\hline & $\widehat{\theta}$ & 2.2729 & 3.1653 & 0.8729 \\
\hline & $\widehat{\gamma}$ & 0.6740 & 0.1478 & 0.0740 \\
\hline \multirow{3}{*}{100} & $\widehat{\alpha}$ & 0.9102 & 0.0132 & 0.0392 \\
\hline & $\widehat{\theta}$ & 1.9558 & 1.7632 & 0.5558 \\
\hline & $\widehat{\gamma}$ & 0.6641 & 0.0972 & 0.0641 \\
\hline \multirow{3}{*}{200} & $\widehat{\alpha}$ & 0.9102 & 0.0072 & 0.0231 \\
\hline & $\widehat{\theta}$ & 1.9558 & 0.8971 & 0.3371 \\
\hline & $\widehat{\gamma}$ & 0.6641 & 0.0596 & 0.0552 \\
\hline \multirow{3}{*}{300} & $\widehat{\alpha}$ & 0.9052 & 0.0055 & 0.0152 \\
\hline & $\widehat{\theta}$ & 1.5979 & 0.4933 & 0.1979 \\
\hline & $\widehat{\gamma}$ & 0.6221 & 0.0440 & 0.0221 \\
\hline \multirow{3}{*}{400} & $\widehat{\alpha}$ & 0.9042 & 0.0039 & 0.0102 \\
\hline & $\widehat{\theta}$ & 1.5445 & 0.3234 & 0.1445 \\
\hline & $\widehat{\gamma}$ & 0.6197 & 0.0310 & 0.0197 \\
\hline \multirow{3}{*}{500} & $\widehat{\alpha}$ & 0.9050 & 0.0031 & 0.0098 \\
\hline & $\widehat{\theta}$ & 1.4891 & 0.1686 & 0.0891 \\
\hline & $\widehat{\gamma}$ & 0.6076 & 0.0255 & 0.0076 \\
\hline \multirow{3}{*}{600} & $\widehat{\alpha}$ & 0.9038 & 0.0026 & 0.0074 \\
\hline & $\widehat{\theta}$ & 1.4714 & 0.1143 & 0.0714 \\
\hline & $\widehat{\gamma}$ & 0.6071 & 0.0217 & 0.0071 \\
\hline \multirow{3}{*}{700} & $\widehat{\alpha}$ & 0.9017 & 0.0020 & 0.0047 \\
\hline & $\widehat{\theta}$ & 1.4594 & 0.0714 & 0.0594 \\
\hline & $\widehat{\gamma}$ & 0.6123 & 0.0161 & 0.0123 \\
\hline \multirow{3}{*}{750} & $\widehat{\alpha}$ & 0.9013 & 0.0019 & 0.0028 \\
\hline & $\widehat{\theta}$ & 1.4400 & 0.0533 & 0.0400 \\
\hline & $\widehat{\gamma}$ & 0.6024 & 0.0148 & 0.0024 \\
\hline
\end{tabular}

$G(x ; a, \alpha, \gamma)=\left(1-e^{-\gamma x^{\alpha}}\right)^{a}, \quad x \geq 0, a, \alpha, \gamma>0$.

(iii) APTW distribution (vi) TW distribution

$G\left(x ; \alpha_{1}, \alpha, \gamma\right)=\frac{\alpha_{1}^{\left(1-e^{-\gamma x^{\alpha}}\right)}-1}{\alpha_{1}-1}, \quad x \geq 0, \alpha_{1}>0, \alpha_{1} \neq 1, \alpha, \gamma>0$.

$$
G(x ; \alpha, \gamma, \lambda)=(1+\lambda)\left(1-e^{-\gamma x^{\alpha}}\right)-\lambda\left(1-e^{-\gamma x^{\alpha}}\right)^{2}, \quad x \geq 0, \alpha, \gamma>0,|\lambda| \leq 1
$$

(v) FWE distribution

$$
G(x ; \alpha, \gamma, \theta)=1-e^{\left.-e^{\gamma x^{2}-\left(\theta / x^{\alpha}\right.}\right)}, \quad x \geq 0, \alpha, \gamma, \theta>0 .
$$

Next, we consider certain analytical measures in order to verify which distribution fits better the considered data.
These analytical measures include (i) discrimination measures such as Akaike information criterion (AIC), Bayesian information criterion (BIC), Hannan-Quinn information criterion (HQIC), consistent Akaike information criterion (CAIC), and (ii) three other goodness-of-fit measures including Anderson-Darling (AD) test statistic, Cramer-von Mises (CM) test statistic, and Kolmogorov-Smirnov (KS) 
TABLE 2: Simulation results of NLTE-W distribution.

\begin{tabular}{|c|c|c|c|c|}
\hline \multicolumn{5}{|c|}{ Set $1: \alpha=0.5, \theta=1.2$, and $\gamma=0.9$} \\
\hline$n$ & Parameters & MLE & MSEs & Biases \\
\hline \multirow{3}{*}{25} & $\widehat{\alpha}$ & 0.5470 & $1.0176 e-02$ & $4.7049 e-02$ \\
\hline & $\widehat{\theta}$ & 1.3739 & $5.7227 e-01$ & $1.7393 e-01$ \\
\hline & $\widehat{\gamma}$ & 1.0060 & $4.6356 e-01$ & 0.1061 \\
\hline \multirow{3}{*}{50} & $\widehat{\alpha}$ & 0.5197 & $3.0224 e-03$ & $1.9750 e-02$ \\
\hline & $\widehat{\theta}$ & 1.3332 & $9.9853 e-02$ & $3.3231 e-02$ \\
\hline & $\widehat{\gamma}$ & 0.9083 & $1.2798 e-01$ & 0.0083 \\
\hline \multirow{3}{*}{100} & $\widehat{\alpha}$ & 0.5053 & $5.9972 e-04$ & $5.3831 e-03$ \\
\hline & $\widehat{\theta}$ & 1.2990 & $5.5752 e-03$ & $-4.4566 e-05$ \\
\hline & $\widehat{\gamma}$ & 0.8908 & $1.8679 e-02$ & -0.0091 \\
\hline \multirow{3}{*}{200} & $\widehat{\alpha}$ & 0.5042 & $4.9037 e-05$ & $5.3695 e-04$ \\
\hline & $\widehat{\theta}$ & 1.2543 & $6.5797 e-05$ & $-6.3885 e-04$ \\
\hline & $\widehat{\gamma}$ & 0.8985 & $4.0895 e-04$ & -0.0014 \\
\hline \multirow{3}{*}{300} & $\widehat{\alpha}$ & 0.5032 & $2.2459 e-06$ & $5.4722 e-05$ \\
\hline & $\widehat{\theta}$ & 1.2398 & $5.7826 e-06$ & $-8.7809 e-05$ \\
\hline & $\hat{\gamma}$ & 0.8998 & $4.0526 e-05$ & -0.0002 \\
\hline \multirow{3}{*}{400} & $\widehat{\alpha}$ & 0.5014 & $3.9207 e-05$ & $2.2864 e-04$ \\
\hline & $\widehat{\theta}$ & 1.2170 & $4.2641 e-05$ & $-2.3844 e-04$ \\
\hline & $\widehat{\gamma}$ & 0.9000 & $6.9245 e-04$ & -0.0009 \\
\hline \multirow{3}{*}{500} & $\widehat{\alpha}$ & 0.5000 & $0.0000 e+00$ & $0.0000 e+00$ \\
\hline & $\hat{\theta}$ & 1.2000 & $0.0000 e+00$ & $0.0000 e+00$ \\
\hline & $\widehat{\gamma}$ & 0.9000 & $0.0000 e+00$ & $0.0000 e+00$ \\
\hline \multirow{3}{*}{600} & $\widehat{\alpha}$ & 0.5000 & $0.0000 e+00$ & $0.0000 e+00$ \\
\hline & $\widehat{\theta}$ & 1.2000 & $0.0000 e+00$ & $0.0000 e+00$ \\
\hline & $\hat{\gamma}$ & 0.9000 & $0.0000 e+00$ & $0.0000 e+00$ \\
\hline \multirow{3}{*}{700} & $\widehat{\alpha}$ & 0.5000 & $0.0000 e+00$ & $0.0000 e+00$ \\
\hline & $\widehat{\theta}$ & 1.2000 & $0.0000 e+00$ & $0.0000 e+00$ \\
\hline & $\widehat{\gamma}$ & 0.9000 & $0.0000 e+00$ & $0.0000 e+00$ \\
\hline \multirow{3}{*}{750} & $\widehat{\alpha}$ & 0.5000 & $0.0000 e+00$ & $0.0000 e+00$ \\
\hline & $\hat{\theta}$ & 1.2000 & $0.0000 e+00$ & $0.0000 e+00$ \\
\hline & $\hat{\gamma}$ & 0.9000 & $0.0000 e+00$ & $0.0000 e+00$ \\
\hline
\end{tabular}

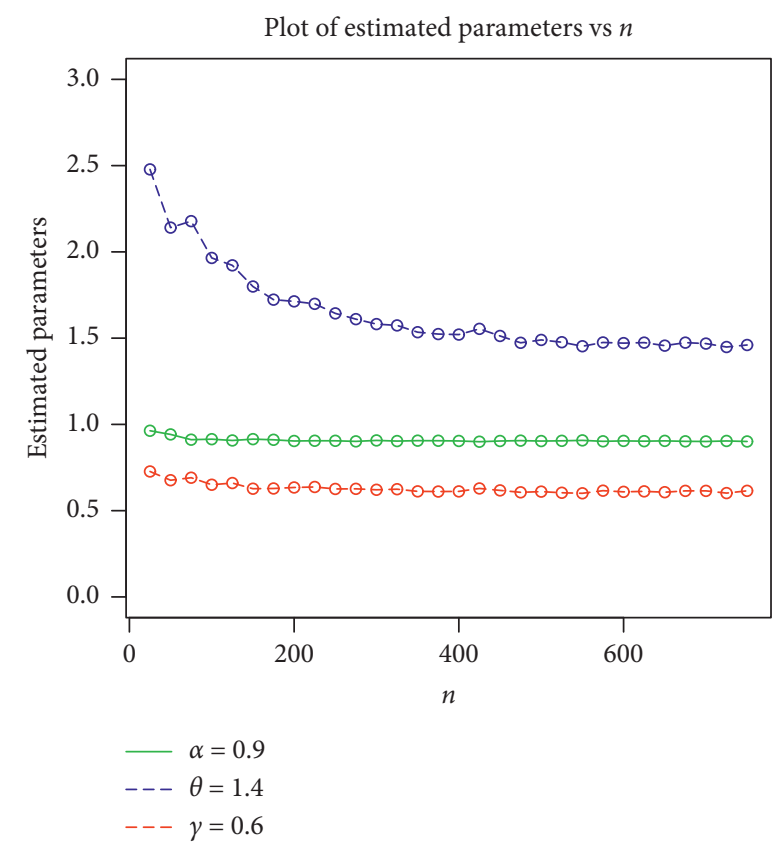

(a)

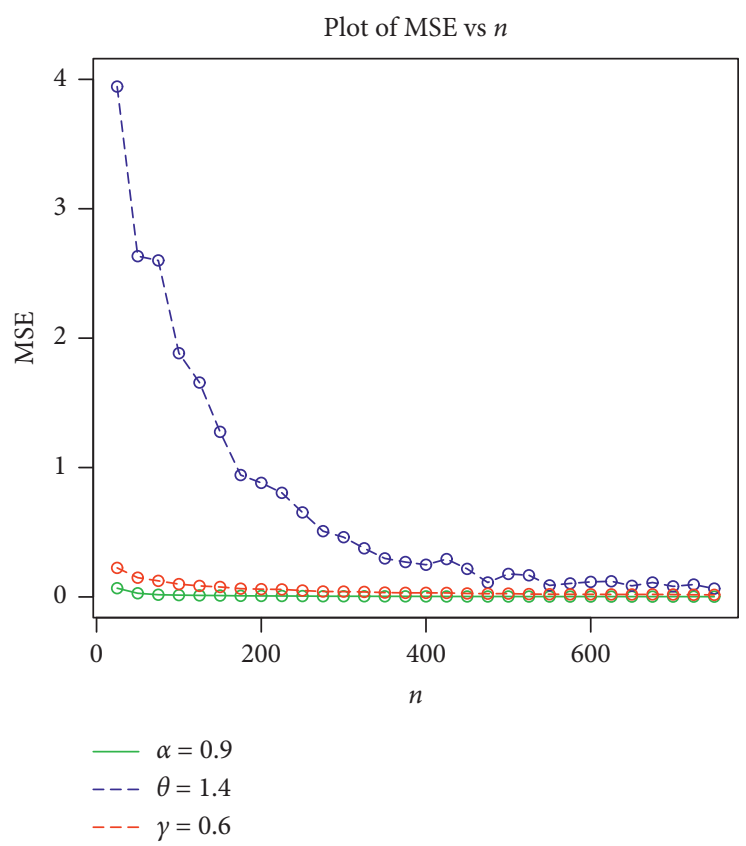

(b)

FIGURE 7: Continued. 


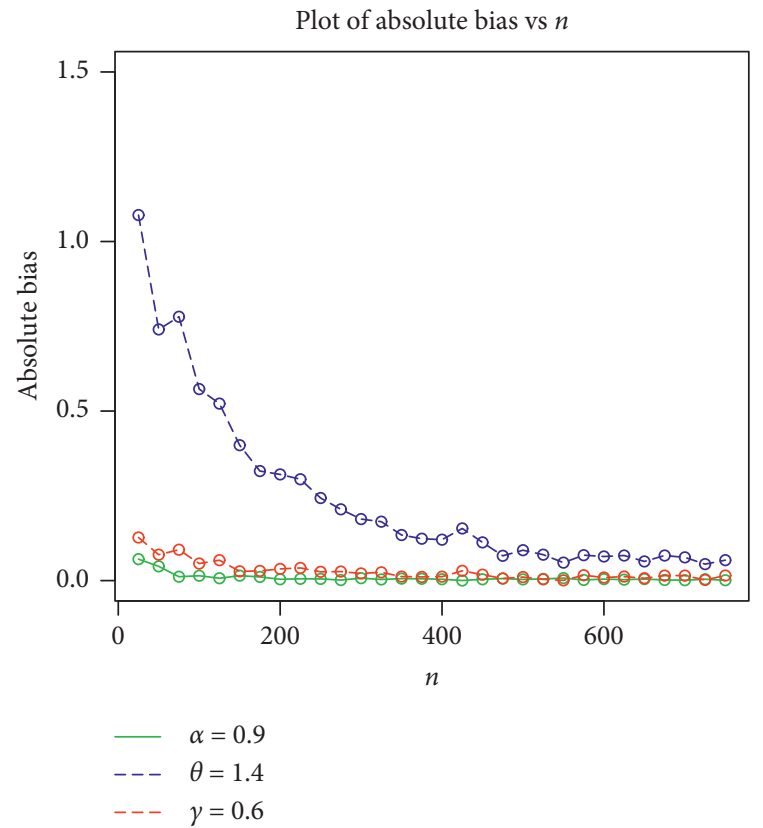

(c)

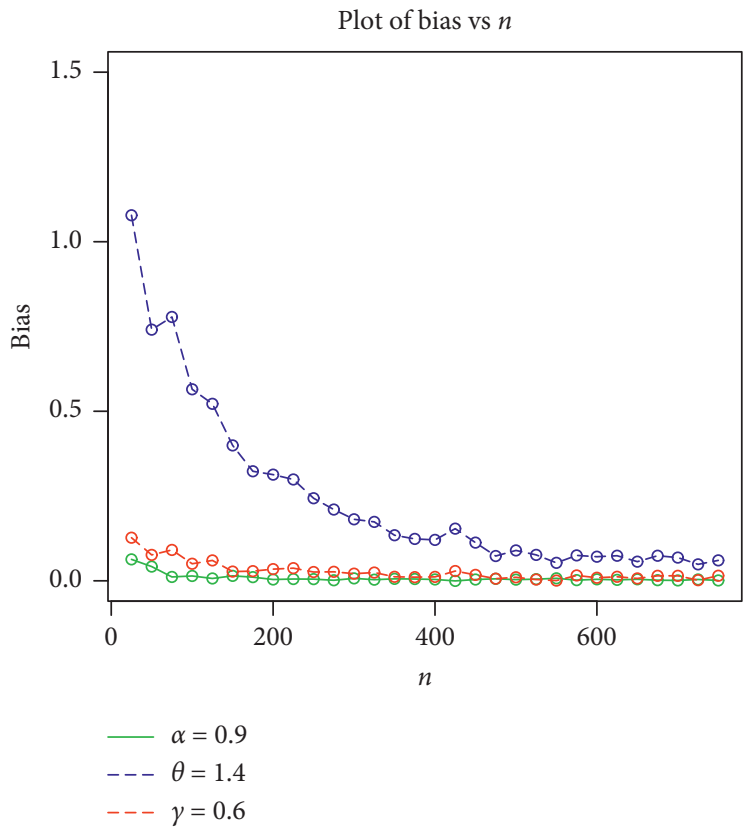

(d)

Figure 7: Plots of MLEs, MSEs, biases, and absolute biases of the NLTE-W model for $\alpha=0.9, \theta=1.4$, and $\gamma=0.6$.

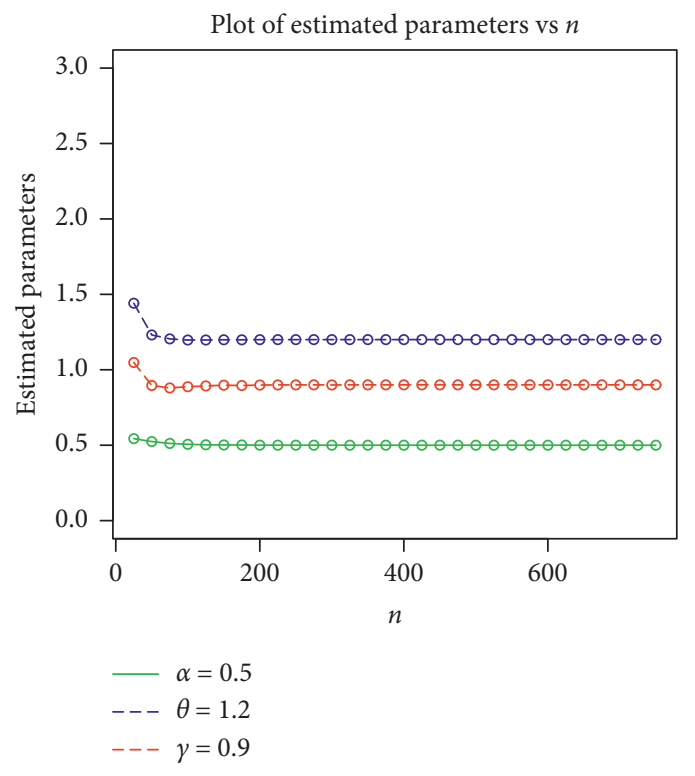

(a)

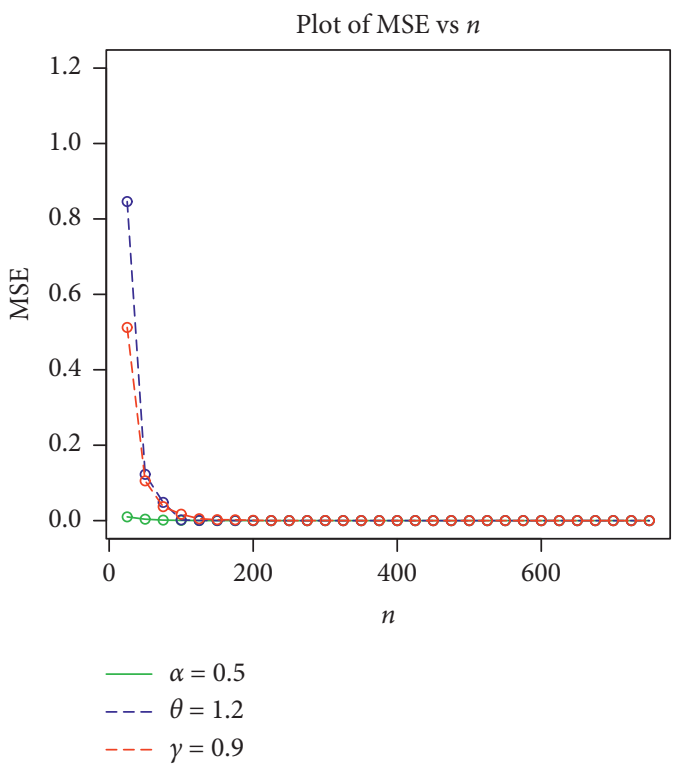

(b)

Figure 8: Continued. 


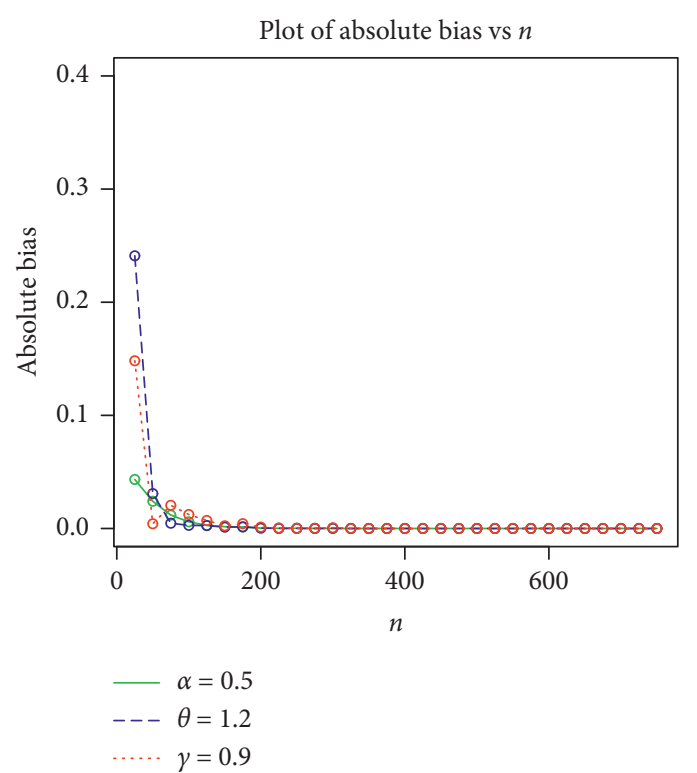

(c)

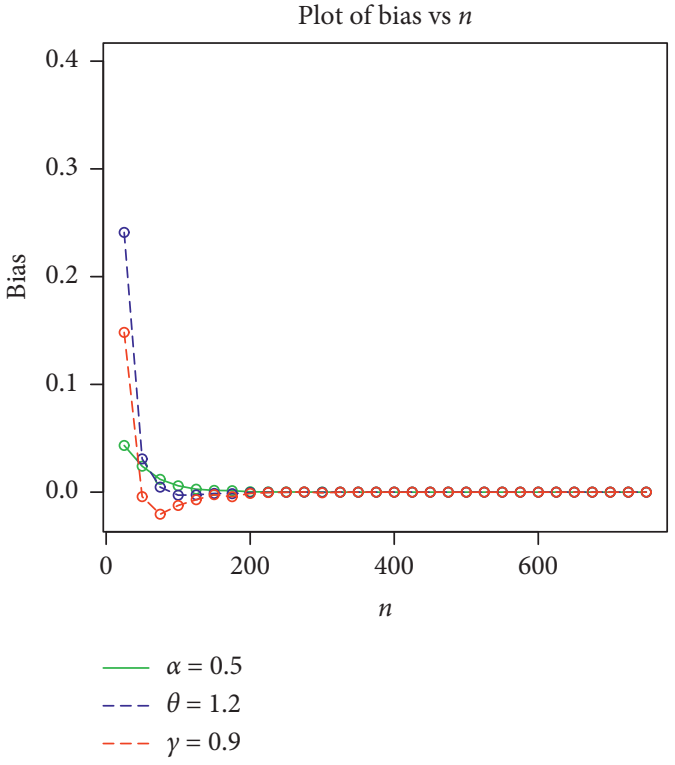

(d)

FIgUre 8: Plots of MLEs, MSEs, biases, and absolute biases of the NLTE-W model for $\alpha=0.5, \theta=1.2$, and $\gamma=0.9$.

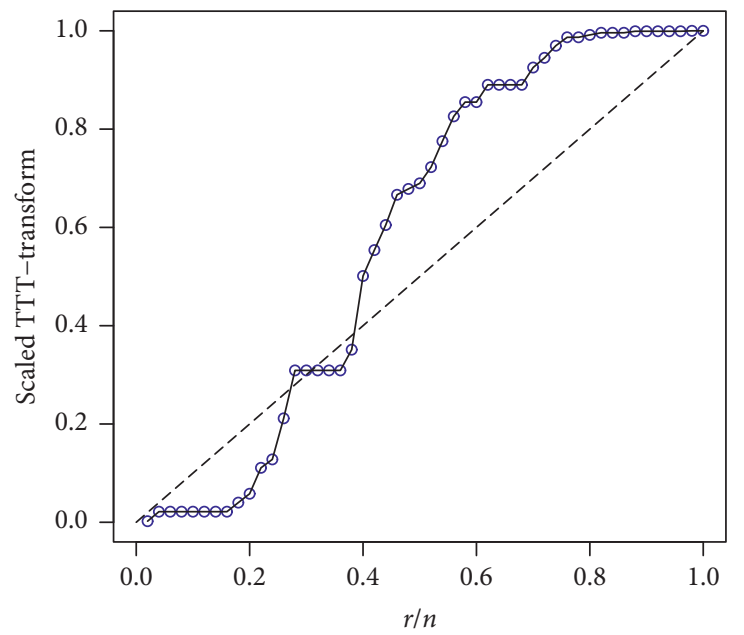

(a)

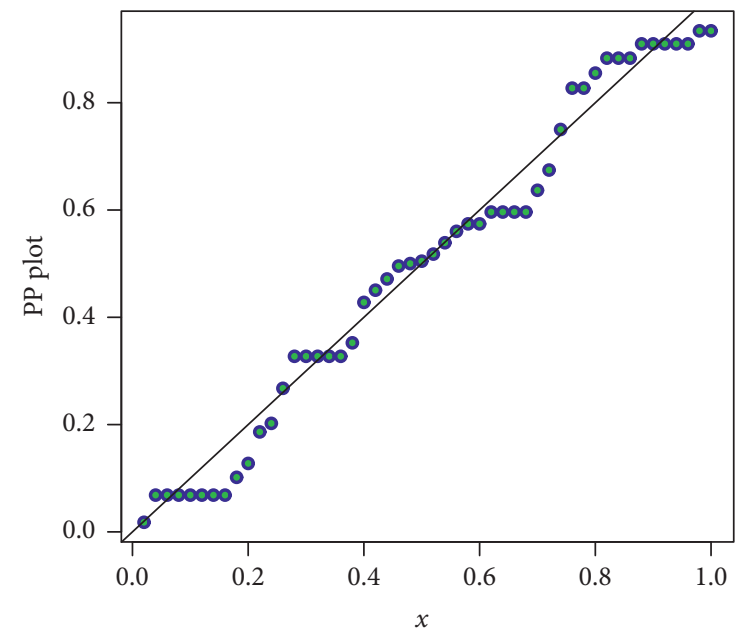

(b)

Figure 9: The scaled TTT-transform and PP plots of the NLTE-W distribution for the Arset data.

TABle 3: The MLEs of the fitted models for data 1.

\begin{tabular}{lccccc}
\hline Dist. & $\hat{\alpha}$ & $\hat{\gamma}$ & $\hat{\theta}$ & $\hat{a}$ & $\hat{\alpha}$ \\
\hline NLTE-W & $0.9340(0.1162)$ & $0.0288(0.0144)$ & $0.0006(0.2288)$ & & \\
Weibull & $0.8759(0.1084)$ & $0.0374(0.0174)$ & & $0.4947(0.0799)$ & $3.8431(3.4375)$ \\
EW & $1.3735(0.7689)$ & $0.0029(0.0010)$ & & & $-0.2919(0.2554)$ \\
APTW & $0.7805(0.1535)$ & $0.0740(0.0564)$ & & & \\
TW & $0.8330(0.1164)$ & $0.0505(0.0274)$ & & & \\
FWE & $0.8549(0.2123)$ & $0.1034(0.6538)$ & $0.9685(1.9543)$ & & \\
\hline
\end{tabular}


TABLE 4: The discrimination measures of the competing models for data 1 .

\begin{tabular}{lcccc}
\hline Dist. & AIC & BIC & CAIC & HQIC \\
\hline NLTE-W & 487.476 & 493.212 & 487.998 & 489.661 \\
Weibull & 492.205 & 496.069 & 492.455 & 493.682 \\
EW & 485.978 & 491.774 & 486.489 & 488.193 \\
APTW & 491.868 & 497.664 & 492.3794 & 494.083 \\
TW & 493.136 & 498.932 & 493.647 & 495.351 \\
FWE & 498.967 & 508.096 & 499.754 & 501.676 \\
\hline
\end{tabular}

TABLE 5: The goodness-of-fit measures of the competing models for data 1.

\begin{tabular}{lcccc}
\hline Dist. & AD & CM & KS & $p$ value \\
\hline NLTE-W & 2.283 & 0.501 & 0.146 & 0.421 \\
Weibull & 3.334 & 0.547 & 0.199 & 0.401 \\
EW & 2.921 & 0.468 & 0.201 & 0.592 \\
APTW & 3.141 & 0.539 & 0.183 & 0.409 \\
TW & 3.246 & 0.530 & 0.188 & 0.407 \\
FWE & 3.476 & 0.598 & 0.201 & 0.386 \\
\hline
\end{tabular}

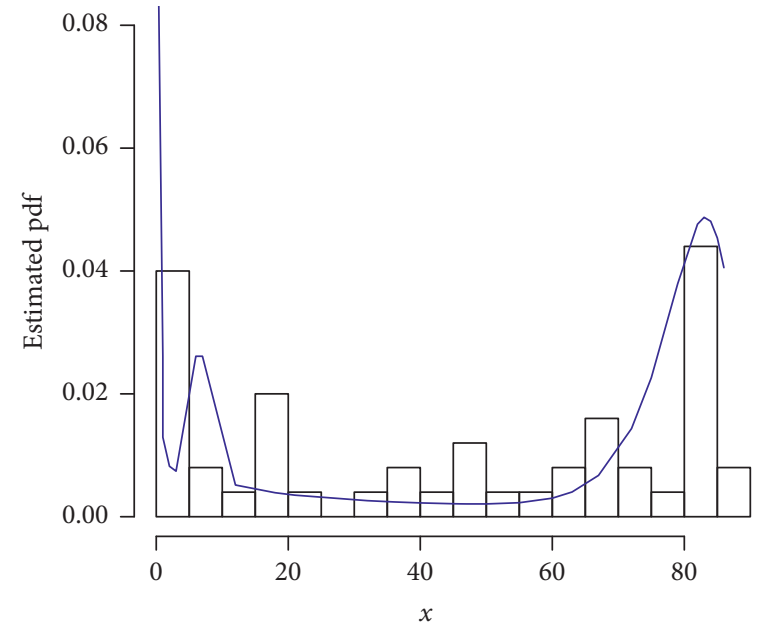

(a)

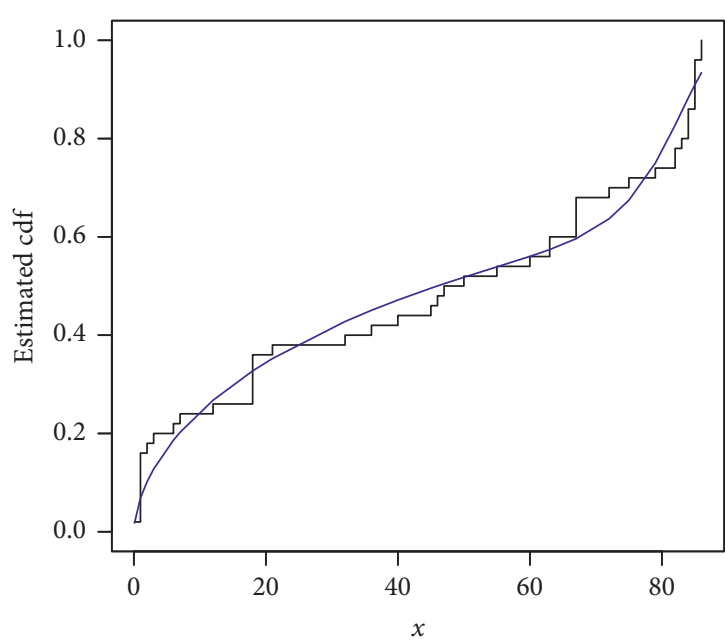

(b)

FIgUre 10: The estimated pdf and cdf of the NLTE-W distribution for the Arset data.

test statistic with corresponding $p$ values; for detail, see Afify et al. [27].

5.1. Arset Data. The first dataset representing the lifetimes of 50 devices taken from Arset [28]. Many authors have analyzed this dataset, including Mudholkar and Srivastava [1]; Xie and Lai [29]; Lai et al. [30]; Sarhan and Zaindin [31]; and Silva et al. [32]. This dataset is known to have a bathtubshaped hazard rate as shown by the scaled TTT-transform plot (Figure 9). For the Arset data, the MLEs with standard errors of the competing models are provided in Table 3. The values of the discrimination measures are provided in $\mathrm{Ta}$ ble 4, whereas the goodness-of-fit measures are presented in Table 5. In support of the results provided in Tables 4 and 5, the fitted pdf and cdf of the proposed model are presented in Figure 10. The scaled TTT-transform and probability- probability (PP) plots of the NLTE-W model are sketched in Figure 9. These plots indicate that the proposed model provides the best fit to data.

5.2. Meeker and Escobar Data. The second data representing the failure times of a sample of 30 devices are taken from Meeker and Escobar [33]. This dataset has already been studied by Almalki and Yuan [34]. The Meeker and Escobar dataset has a bathtub-shaped hazard function as indicated by the scaled TTT-transform plot (Figure 11). Corresponding to the second data, the MLEs with standard errors of the competing models are provided in Table 6 . The values of the discrimination measures are provided in Table 7 , whereas the goodness-of-fit measures are presented in Table 8. In support of the results provided in Tables 7 and 8, the fitted pdf and cdf of the proposed model are presented in 


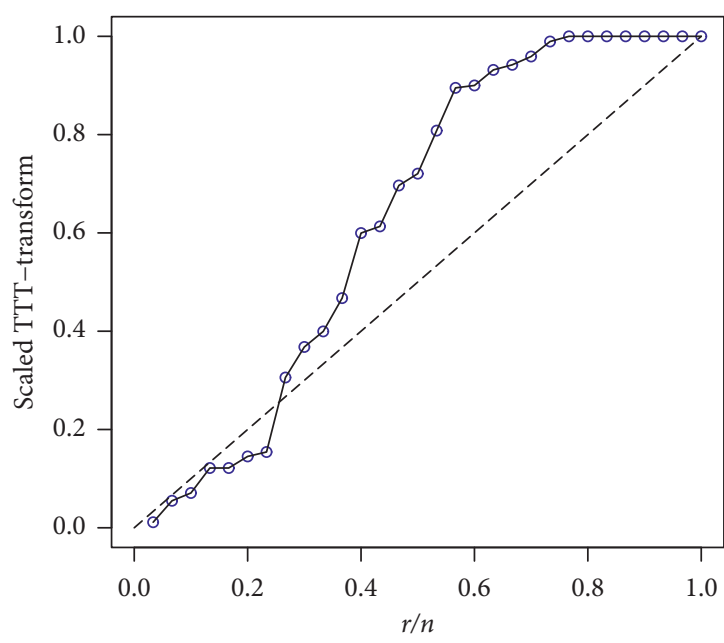

(a)

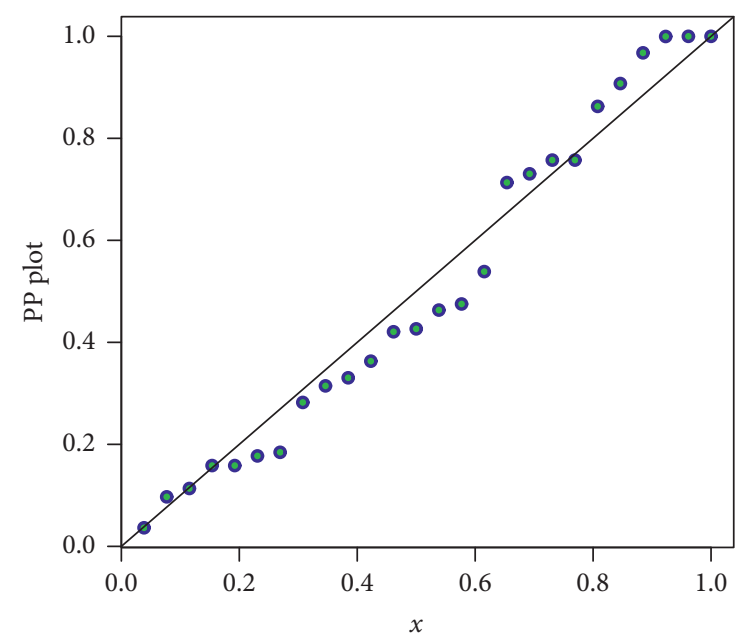

(b)

FIgURE 11: The scaled TTT-transform and PP plots of the NLTE-W distribution for the Meeker and Escobar data.

TABLE 6: The MLEs of the fitted models for data 2.

\begin{tabular}{lccccc}
\hline Dist. & $\hat{\alpha}$ & $\hat{\gamma}$ & $\hat{\theta}$ & $\hat{a}$ & $\hat{\alpha_{1}}$ \\
\hline NLTE-W & $1.1766(0.0439)$ & $0.0929(0.2735)$ & $0.0021(0.0003)$ & & \\
Weibull & $1.0931(0.0734)$ & $0.0034(0.0012)$ & & $1.0763(0.2522)$ & \\
EW & $0.0037(0.0017)$ & $1.0865(0.0807)$ & & & \\
APTW & $1.0475(0.1464)$ & $0.0061(0.0053)$ & & & $-0.3995(3.8457)$ \\
TW & $1.1953(0.0435)$ & $0.0022(0.0065)$ & & \\
FWE & $1.864(0.7463)$ & $0.0765(0.9675)$ & $9.7547(3.8654)$ & & \\
\hline
\end{tabular}

TABLE 7: The discrimination measures of the competing models for data 2.

\begin{tabular}{lcccc}
\hline Dist. & AIC & BIC & CAIC & HQIC \\
\hline NLTE-W & 364.932 & 369.135 & 365.855 & 366.277 \\
Weibull & 373.409 & 376.212 & 373.854 & 374.306 \\
EW & 375.498 & 379.702 & 376.422 & 376.843 \\
APTW & 373.533 & 377.737 & 374.456 & 374.878 \\
TW & 374.047 & 378.251 & 374.970 & 375.392 \\
FWE & 383.754 & 394.734 & 384.8796 & 390.569 \\
\hline
\end{tabular}

TABLE 8: The goodness-of-fit measures of the competing models for data 2.

\begin{tabular}{lcccr}
\hline Dist. & AD & CM & KS & $p$ value \\
\hline NLTE-W & 1.229 & 0.235 & 0.165 & 0.783 \\
Weibull & 1.873 & 0.314 & 0.224 & 0.572 \\
EW & 1.877 & 0.315 & 0.201 & 0.592 \\
APTW & 1.766 & 0.291 & 0.221 & 0.104 \\
TW & 1.784 & 0.295 & 0.212 & 0.133 \\
FWE & 2.087 & 0.395 & 0.298 & 0.138 \\
\hline
\end{tabular}

Figure 12. The scaled TTT-transform and probabilityprobability (PP) plots of the NLTE-W model are sketched in Figure 11. It is clear in Figure 12 that the NLTE-W distribution fits the left and right peaks in the histogram better (see the fitted pdf) and its survival function follows the estimated cdf closely.

From the results provided in Tables 4 and 5 (for data 1) and Tables 7 and 8 (for data 2), it is clear that the 


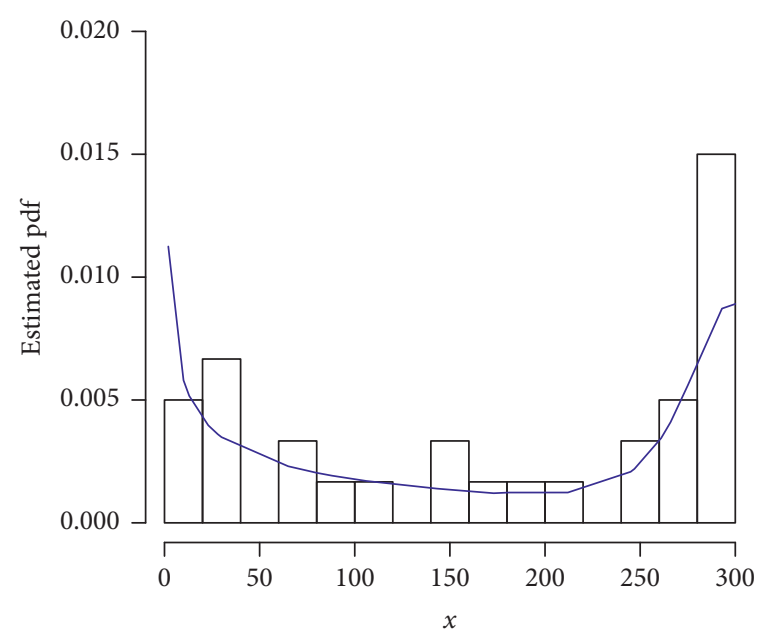

(a)

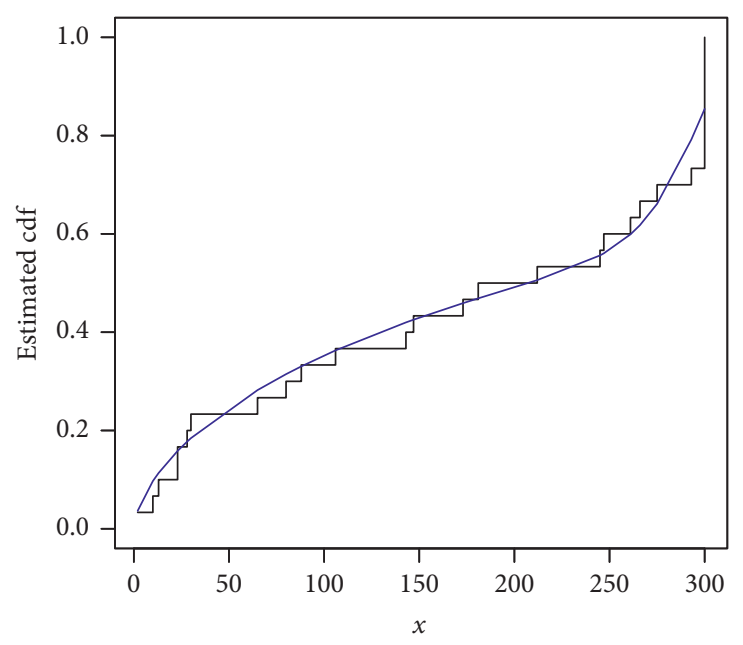

(b)

FIgure 12: The estimated pdf and cdf of the NLTE-W distribution for the Meeker and Escobar data.

proposed model could be chosen as the best model among the fitted models since the proposed model has the lowest values of the goodness-of-fit measures and maximum $p$ value.

\section{Concluding Remarks}

A new family of distributions called a new lifetime exponential- $X$ family has been introduced. A three-parameter special submodel of the proposed family called a new lifetime exponential-Weibull distribution is studied in detail. The proposed model is very flexible and is capable of accommodating different shapes of the failure rates including bathtub-shaped to describe the failure behavior of a variety of lifetime datasets. Maximum-likelihood estimators of the model parameters are obtained and a Monte Carlo simulation study has been carried out. Finally, two real datasets with bathtub-shaped failure rate, as indicated by the scaled TTT-transform plots, have been analyzed for illustrative purposes.

Future work includes MCMC methods with censored data, modeling regression problems with covariates, and parameter reduction.

\section{Data Availability}

The data used to support the findings of this study are included within the article.

\section{Conflicts of Interest}

The authors declare that there are no conflicts of interest regarding the publication of this paper.

\section{Acknowledgments}

The first author also acknowledges the support of 2019 Soft Science Project of Science and Technology Department of Jilin Province, Research on Service Quality Improvement of
Science and Technology Innovation Platform of Jilin Province (no. 20190601051FG).

\section{References}

[1] G. S. Mudholkar and D. K. Srivastava, "Exponentiated Weibull family for analyzing bathtub failure-rate data," IEEE Transactions on Reliability, vol. 42, no. 2, pp. 299-302, 1993.

[2] M. Xie, Y. Tang, and T. N. Goh, "A modified Weibull extension with bathtub-shaped failure rate function," Reliability Engineering \& System Safety, vol. 76, no. 3, pp. 279-285, 2002.

[3] T. Zhang and M. Xie, "On the upper truncated Weibull distribution and its reliability implications," Reliability Engineering \& System Safety, vol. 96, no. 1, pp. 194-200, 2011.

[4] M. Bebbington, C.-D. Lai, and R. Zitikis, "A flexible Weibull extension,” Reliability Engineering \& System Safety, vol. 92, no. 6, pp. 719-726, 2007.

[5] Z. Ahmad, "The Zubair-G family of distributions: properties and applications," Annals of Data Science, vol. 7, no. 2, pp. 195-208, 2018.

[6] Z. Ahmad, M. Ilyas, and G. G. Hamedani, "The extended alpha power transformed family of distributions: properties and applications," Journal of Data Science, vol. 17, no. 4, pp. 726-741, 2019.

[7] Z. Ahmad, M. Elgarhy, and N. Abbas, "A new extended alpha power transformed family of distributions: properties and applications," Journal of Statistical Modelling: Theory and Applications, vol. 1, no. 1, pp. 13-28, 2019.

[8] H. P. Zhu, X. Xia, H. Y. Chuan, A. Adnan, S. F. Liu, and Y. K. Du, "Application of Weibull model for survival of patients with gastric cancer," BMC Gastroenterology, vol. 11, no. 1, pp. 1-15, 2011.

[9] S. J. Al-Malki, "Statistical analysis of lifetime data using new modifed weibull distributions," The University of Manchester, Manchester, UK, Doctoral dissertation, 2014.

[10] A. S. Wahed, T. M. Luong, and J.-H. Jeong, "A new generalization of Weibull distribution with application to a breast cancer data set," Statistics in Medicine, vol. 28, no. 16, pp. 2077-2094, 2009. 
[11] D. R. Cox, "Regression models and life-tables," Journal of the Royal Statistical Society: Series B (Methodological), vol. 34, no. 2, pp. 187-202, 1972.

[12] L. Bagnato and A. Punzo, "Finite mixtures of unimodal beta and gamma densities and the $\mathrm{k}$-bumps algorithm," Computational Statistics, vol. 28, no. 4, pp. 1571-1597, 2013.

[13] S. A. Bakar, N. Hamzah, M. Maghsoudi, and S. Nadarajah, "Modeling loss data using composite models," Insurance: Mathematics and Economics, vol. 61, pp. 146-154, 2015.

[14] A. Punzo, L. Bagnato, and A. Maruotti, "Compound unimodal distributions for insurance losses," Insurance: Mathematics and Economics, vol. 81, pp. 95-107, 2018.

[15] A. Punzo, A. Mazza, and A. Maruotti, "Fitting insurance and economic data with outliers: a flexible approach based on finite mixtures of contaminated gamma distributions," Journal of Applied Statistics, vol. 45, no. 14, pp. 2563-2584, 2018.

[16] E. Brito, G. M. Cordeiro, H. M. Yousof, M. Alizadeh, and G. O. Silva, "The Topp-Leone odd log-logistic family of distributions," Journal of Statistical Computation and Simulation, vol. 87, no. 15, pp. 3040-3058, 2017.

[17] H. M. Yousof, A. Z. Afify, G. G. Hamedani, and G. Aryal, "The burr X generator of distributions for lifetime data," Journal of Statistical Theory and Applications, vol. 16, no. 3, pp. 288-305, 2017.

[18] F. Merovci, M. Alizadeh, H. M. Yousof, and G. G. Hamedani, "The exponentiated transmuted-G family of distributions: theory and applications," Communications in StatisticsTheory and Methods, vol. 46, no. 21, pp. 10800-10822, 2017.

[19] G. M. Cordeiro, H. M. Yousof, T. G. Ramires, and E. M. M. Ortega, "The burr XII system of densities: properties, regression model and applications," Journal of Statistical Computation and Simulation, vol. 88, no. 3, pp. 432-456, 2018.

[20] G. G. Hamedani, E. Altun, M. Ç. Korkmaz, H. M. Yousof, and N. S. Butt, "A new extended G family of continuous distributions with mathematical properties, characterizations and regression modeling," Pakistan Journal of Statistics and Operation Research, vol. 14, no. 3, pp. 737-758, 2018.

[21] D. G. G. Hamedani, H. M. Yousof, M. Rasekhi, M. Alizadeh, and S. M. Najibi, "Type I general exponential class of distributions," Pakistan Journal of Statistics and Operation Research, vol. 14, no. 1, pp. 39-55, 2018.

[22] G. G. Hamedani, M. Rasekhi, S. Najibi, H. M. Yousof, and M. Alizadeh, "Type II general exponential class of distributions," Pakistan Journal of Statistics and Operation Research, vol. 15, no. 2, pp. 503-523, 2019.

[23] A. Alzaatreh, C. Lee, and F. Famoye, "A new method for generating families of continuous distributions," Metron, vol. 71, no. 1, pp. 63-79, 2013.

[24] Z. Ahmad, G. G. Hamedani, and N. S. Butt, "Recent developments in distribution theory: a brief survey and some new generalized classes of distributions," Pakistan Journal of Statistics and Operation Research, vol. 15, no. 1, pp. 87-110, 2019.

[25] F. Jamal and M. Nasir, "Some new members of the T-X family of distributions," in Proceedings of the 17th International Conference on Statistical Sciences, Lahore, Pakistan, January 2019.

[26] Z. Ahmad, E. Mahmoudi, S. Dey, and S. K. Khosa, "Modeling vehicle insurance loss data using a new member of T-X family of distributions," Journal of Statistical Theory and Applications, vol. 19, no. 2, pp. 133-147, 2020.
[27] A. Z. Afify, G. M. Cordeiro, H. M. Yousof, A. Saboor, and E. M. Ortega, "The Marshall-Olkin additive Weibull distribution with variable shapes for the hazard rate," Hacettepe Journal of Mathematics and Statistics, vol. 47, no. 2, pp. 365-381, 2018.

[28] M. V. Aarset, "How to identify a bathtub hazard rate," IEEE Transactions on Reliability, vol. R-36, no. 1, pp. 106-108, 1987.

[29] M. Xie and C. D. Lai, "Reliability analysis using an additive Weibull model with bathtub-shaped failure rate function," Reliability Engineering \& System Safety, vol. 52, no. 1, pp. 87-93, 1996.

[30] C. D. Lai, M. Xie, and D. N. P. Murthy, "A modified Weibull distribution," IEEE Transactions on Reliability, vol. 52, no. 1, pp. 33-37, 2003.

[31] A. M. Sarhan and M. Zaindin, "Modifed Weibull distribution. APPS," Applied Sciences, vol. 11, pp. 123-136, 2009.

[32] G. O. Silva, E. M. M. Ortega, and G. M. Cordeiro, "The beta modified Weibull distribution," Lifetime Data Analysis, vol. 16, no. 3, pp. 409-430, 2010.

[33] W. Q. Meeker and L. A. Escobar, Statistical Methods for Reliability Data, John Wiley \& Sons, Hoboken, NY, USA, 2014.

[34] S. J. Almalki and J. Yuan, "A new modified Weibull distribution,” Reliability Engineering \& System Safety, vol. 111, pp. 164-170, 2013. 